\title{
Safety of Growth Hormone Therapy in Patients with Idiopathic Short Stature: A Systematic Review
}

\author{
Aaron Trubian${ }^{1}$, Isabelle Bravo Ribeiro Cavassa ${ }^{1}$, Heloisa Rodrigues Alves Bobato ${ }^{2}$, Roberto Zonato \\ Esteves $^{3}$ and Elaine Rossi Ribeiro ${ }^{3 *}$ \\ ${ }^{1}$ Medical students at Pequeno Príncipe Faculties, Brazil \\ ${ }^{2}$ Student of the Master's Degree Program in Health Sciences Teaching, Brazil \\ ${ }^{3}$ Faculty researcher at Pequeno Príncipe Faculties, Brazil
}

\begin{abstract}
Introduction: Idiopathic short stature is a common condition in the pediatric population requiring growth hormone therapy. Thus, based on patient safety concepts, we sought to group the main literature data related to this aspect in different countries.

Objectives: The aim of this systematic review was to identify what the literature says about the main safety aspects of growth hormone therapy in children with idiopathic short stature.
\end{abstract}

Methods: This is a systematic review using PubMed, BVS portal, Cochrane, LILACS, and CAPES databases, with the descriptors idiopathic short stature, human growth hormone therapy, patient safety, children, and the boolean "AND" between them. Inclusion criteria were children with idiopathic short stature treated at some point during their lifetime with growth hormone and studies dating between 2015 and 2020.

Results: The safety of growth hormone therapy in this patient profile remained consistent with data previously described in the literature. There was a difference in the incidence of adverse effects, indicating that there are various degrees of resistance to IGF-1 action in these individuals, and the response to GH may vary in this heterogeneous population. These children need higher doses of GH to get the same benefits compared to other etiologies of short stature.

Conclusion: Adverse effects were mostly mild, with rare severe cases, thus, it can be concluded that the use of GH in children with idiopathic short stature has an efficient safety profile, although further long-term studies are required.

KEYWORDS: Idiopathic short stature; Growth hormone therapy; Patient safety; Children

ABBREVIATIONS: NPIG: Children Born Small for Gestational Age; GHD: Growth Hormone Deficiency; ISS: Idiopathic Short Stature; PNSP: National Program for Patient Safety

\section{INTRODUCTION}

The iconic timeless proposition made by Hippocrates "first, do no harm" can still be used nowadays. For a long time, medical errors were viewed as an adversity by hospitals. However, in late 1999, the

report "To Err is Human" was published by the Institute of Medicine (IOM) and estimated that 44,000 to 98,000 Americans die annually because of healthcare-related errors. Since the 2000s the term "patient safety" has entered the scope of worldwide discussion, and in this sense, in 2004, the World Health Organization (WHO)
Quick Response Code:

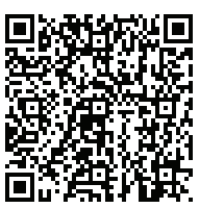

Address for correspondence: Elaine Rossi Ribeiro, Faculty researcher at Pequeno Príncipe Faculties, Brazil

Received: July 31, $2021 \quad$ Published: August 09, 2021

How to cite this article: Aaron T, Isabelle BRC, Heloisa RAB, Roberto ZE, Elaine RR. Safety of Growth Hormone Therapy in Patients with Idiopathic Short Stature: A Systematic Review. 20213(4) OAJBS.ID.000314. DOI: 10.38125/OAJBS.000314 
established the World Alliance for Patient Safety to identify global priorities in this area [1]. Qualified health care is the goal of all services and professionals, therefore, patient safety in different aspects is essential for them to receive carefree of harm1. For such, it is understood that a relevant area for research in this sense would be endocrinology and, in this case, specifically regarding growth hormone therapy in patients with idiopathic short stature.

Growth consists of increasing body size and is a physiological process in which cell multiplication and increment occur. Each individual has a genetic potential for growth, which depends on genetic, metabolic, and endocrinological factors, lifestyle habits, diet, physical activity, and sleep. When the expected potential is not reached or shows that it will not occur, it is possible to characterize short stature [2]. According to Yadav [3], there are two main approaches for detecting short stature:

a) Single point measurement: parameter defined as height below-2 standard deviation (SD) for age and sex and population or height below 2 SD of mean family height.

b) Serial growth monitoring: growth impairment in this parameter is defined as crossing below two percentiles on the growth curve compatible for age and sex of the child, in this sense, all children with height below the 3rd percentile on the growth curve specific for gender and population deserve investigation.

Several causes of growth deficits are identifiable in children. There are, however, some children with normal growth hormone levels and no evidence of endocrine, chromosomal nutritional, or systemic disorders that would justify their short stature. Thus, these individuals are commonly diagnosed under the spectrum of idiopathic short stature (ISS); [4]. According to CHILD5, the approximate prevalence is estimated to be 16 per 1,000 children.

Since ISS is a diagnosis of exclusion based on axiological parameters, these children are described with a height of more than 2 SD below the average for age, sex and population, healthy, with normal proportions at birth, without evident systemic, genetic or endocrine disorders [4-6].

The explanation for this fact is due to the heterogeneous characteristic of the group, and to subtle disturbances in GH secretion and sensitivity and a combination of genetic factors that influence the physiology of the growth plate.

In this sense, the etiology of idiopathic short stature is complex and may be related to various potential factors, including disturbances in pituitary secretion, low physical activity, mutations in the GH receptor gene, disordered hormone signaling, reduced sensitivity, changes in the concentrations of IGF carrier proteins (IGFBPs) - there is six of them - or decreased sensitivity to IGF [7]. Growth hormone therapy is considered safe in the literature, and the occurrence of serious adverse effects is rare [5,8].

However, some issues raised, noting increased cause-specific mortality in young adults who received GH in childhood, renew concerns about the long-term safety of the therapy $[5,8,9]$. The GH treatment was approved by the Food and Drug Administration (FDA) in 2003 for children presenting with height score (SDS) $\leq-2.25$ SDS $(\leq 1.2$ percentile), thus, recommending a therapeutic dose of 0.30-0.37 mg rhGH - (kg - week) and subsequently the therapy was approved in Canada and other countries, but not in the European Union and Japan [5]. At present, there is some dispute about the efficacy and safety of long-term GH treatment for children with idiopathic short stature. Although there are concerns and some negative associations, the safety results observed in various studies in different countries were favorable, and the studies with a higher level of evidence seem to result in more consistent outcomes, demonstrating a good safety profile of GH treatment in these patients. Given this scenario, it is justified the acknowledgment regarding patient safety aspects may be related to the use of $\mathrm{GH}$, a question that guided the objective of this study: to identify what the literature highlights about the main safety aspects of growth hormone therapy in patients with idiopathic short stature.

\section{METHODS}

This is a predominantly qualitative systematic review without meta-analysis. The review was carried out according to the Cochrane Collaboration and PRISMA - Preferred Reporting Items for Systematic Review and Meta-analysis Statement recommendations [10].

The electronic databases used for the search strategy were PubMed, Portal da CAPES, Portal da Biblioteca Virtual de Saúde (BVS), Lilacs and Chocrane. The descriptors used to answer the question "What does the literature expose about the main aspects related to patient safety and growth hormone therapy in children with idiopathic short stature? " were based on the PICO acronym and guided the search strategy, these are being: idiopathic short stature AND growth hormone therapy AND patient safety and their English language correspondents are "patient safety, "growth hormone treatment", "idiopathic short stature"; "children". The term idiopathic short stature was not found indexed in MESH/DECS.

The search included scientific articles published between 2015 and 2020, and the criteria of Downs and Black11 were used as a tool to assess the methodological quality of each study. Through a questionnaire containing 27 questions, divided into four groups: presentation (evaluates items such as clarity in the description of objectives, confounding variables, probability values); external validity (related to the extrapolation of data to the population from which the sample was planned); internal validity (analysis of biases, reliability of exposure and outcome measures, and use of confounding variables); and power of the study, we obtained an average score of 12 to 14 points, which ensures a good quality to systematize the evidence from each study.

The protocol of this review was submitted to PROSPERO -International prospective register of systematic review - on September 26, 2020 and can be identified by the number CRD42020206298

\section{RESULTS}

For the elaboration of this study, 14 articles were analyzed, 5 of which were produced in Korea, 3 in the United States of America, 3 in China, 1 in France, 1 in the United Kingdom, and 1 in Saudi Arabia. The year that had the most publications about the topic was 2018, with 6 articles, followed by 2020 with 4 articles, in sequence, in 2015 and 2019 there were 2 articles per year. To facilitate the understanding of the results, the analyzed content was pedagogically subdivided into 3 main categories: patient profile, treatment, and adverse effects.

\section{Patient Profile: Population and Diagnostic Criteria}

Most of the samples were pre-pubescent children, with the average age range in girls being 4 to 9 years, and boys 4 to 11 years. The main inclusion criteria for idiopathic short stature were related to normal thyroid function, GH stimulation test values above 10 
$\mathrm{mg} / \mathrm{mL}$, absence of previous GH treatment, and absence of any other known etiology leading to short stature. Furthermore, in the studies reviewed, we had a diversity of patients, such as those with GHD and children born small for gestational age [7,8,11-13].

These differential diagnoses were of great value to our analysis, as they made it possible to compare the safety of growth hormone from different conditions to our population of interest. The GHD diagnostic criteria were individuals diagnosed in infancy or at least 1 year before the study, with height below -2DP or annual growth velocity was below -1DP, and defective GH response on provocative testing (below $10 \mu \mathrm{g} / \mathrm{L}$ ) associated with an IGF1-score <-2DP NPIG patients were those who were born smaller than normal for gestational age, below the 10th percentile. We also emphasize that the definition and standards for idiopathic short stature differ in the protocols of each country [5].

\section{Treatment: Dosage, Formulations and Combinations}

The dosage regimens instituted were extremely similar, so that the authors used doses that varied according to the FDA 2003 $[5,8,9,12-18]$ or the Chinese society of pediatric endocrinology $[4,6,7,16,19]$ which recommend doses of $0.30-0.37 \mathrm{mg} / \mathrm{kg} /$ week and $0.35-0.46 \mathrm{mg} / \mathrm{kg} /$ week, respectively. The therapeutic administration in the studies was subcutaneous once a day for 6 to 7 days bedtime. It should be noted that there were slight variations in dosages, formulations, and combination with other hormones among the different investigators.

The safety endpoints included analysis of daily formulation rhGH products, weekly formulation rhGH product LB0300217, and included analysis of the combination of daily rhGH with a GNRH agonist leuprorelin 18.

\section{Safety Outcomes: Side Effects}

The pattern of analysis of safety outcomes adopted contained similar evaluation parameters, with slight variations in the observation of side effects due to different therapeutic regimens used by the authors and their respective objectives. In this sense, the most uniform metrics to evidence patient safety were adverse effects (AE), supported on the registration system for safety data analysis, MedDRA, and defined essentially according to the clinical and laboratory data acquired. The main parameters were, global clinical assessment, IGF-1 and IGFBP-3 levels, glycemic assessment - fasting glucose, glycated hemoglobin, baseline-fasting insulin levels, thyroid function, blood biochemistry, bone age, bone density, infectious screening, application site reactions.

The main side effects were infections, especially related to the upper respiratory tract, tonsillar hypertrophy, local reactions and pain at the application site. Metabolic and hormonal changes such as hyperglycemia, hyperinsulinemia, glucose intolerance states, hypothyroidism and gynecomastia. In the study by Hou [7] when comparing GHD and ISS children found hyperinsulinemia states were observed more frequently occurring in 23 ISS patients, 16 $(10.7 \%)$ in the first year, were considerably higher $(\mathrm{p}<0.05)$, suggesting that they are more prone to this phenomenon. Water and sodium retention, hypertension, spontaneous benign intracranial hypertension, hydrocele, musculoskeletal changes such as arthralgia, myalgia, bone fractures, scoliosis, neoplasms, cardiovascular and cerebrovascular diseases.

Although some rare serious events were observed, the final result of the analysis the therapy was proven to be safe in our population of interest. The safety profile of LB03002 at doses of
$0.5 \mathrm{mg} / \mathrm{kg} /$ week and $0.7 \mathrm{mg} / \mathrm{kg} /$ week for 26 weeks demonstrated results consistent with those observed in studies using daily rhGh, but further long-term evaluations of this formulation are needed. The only adverse effects noted were injection site reactions (heat, erythema, and swelling).

Therefore, it is inferred that the drug studied holds great potential to become an option in therapeutic arsenal used to treat these children because in theory, it adds greater convenience and consequently greater adherence to treatment. Additionally, it holds potential economic value, since children with idiopathic short stature need higher doses of hormone to obtain the same benefits compared to other etiologies of short stature such as GHD children7. In addition, individualized therapy regimens such as those performed by Counts14, also offer a financial advantage with a good safety profile. The studies using the daily rhGH formulation proposed by both societies the safety endpoints behaved in line with previous publications and no new concerns about the therapy emerged.

However, according to the Benabbad study18, it can be evidenced that in general there is no robust evidence for the safety of the institution of GnRH agonists in conjunction with injections of daily formulation of growth hormone since there were more adverse reactions in the combination group, with more events of bone fractures, gynecomastia, and scoliosis than in the control group. With different doses, there were different prevalence's of side effects, indicating different responses to growth hormone in this group. Most were mild and the occurrence of serious adverse effects was rare, a result consistent with what has been previously observed in the literature.

\section{CONCLUSION}

Based on the extracted information, the evidence points out that the group of children listed with idiopathic short stature heterogeneously presents in the international literature, due to their similarities with other conditions, so that criteria may vary between different countries and authors. Children with idiopathic short stature due to similarities shared with other conditions such as Prader-Willi syndrome and NPIG have reduced sensitivity to somatotrophic action. Thus, they require higher therapeutic doses to obtain the same benefits compared to other groups with defined short stature etiologies, evidencing higher treatment costs. The safety profile of GH used in different doses, products, therapeutic regimens, and associations was proved to be generally safe.

The main limitations of this review permeate the time factor, the heterogeneity of some samples, few randomized clinical trials with the primary objective for the analysis of safety outcomes, in addition none of the studies works the psychological and emotional aspects of treated children that in theory are also part of the larger concept of patient safety. Furthermore, questions have been raised about the ideal dose, and there is no reliable information for certain therapeutic regimes and long-term formulations.

In this sense, the treatment, despite being safe, must include in its structure consideration of factors related to functionality and individuality of patients, as well as evaluations related to relevant adverse effects, through systematic clinical monitoring, especially in children with a personal or family history of endocrinological metabolic disorders or neoplasms, and further research should be conducted in order to clarify the nuances related to the safety of long-term therapy in this select group. 


\section{REFERENCES}

1. Reis CT, Martins M, Laguardia J (2013) Patient safety as a dimension of the quality of health care - A look at the literature. Cien Saude Colet 18(7): 2029-2036.

2. Brasil (2012) Ministério da Saúde. Saúde da criança: crescimento e desenvolvimento. Cadernos de Atenção Básica 33: 271.

3. Yadav S, Dabas A (2015) Approach to short stature. Indian J Pediatr 82(5): 462-470.

4. Chung WY, Yoo HW, Hwang JS, Ko CW, Kim HS, et al. (2018) Effect of growth hormone therapy on height velocity in korean children with idiopathic short stature: A Phase III randomized controlled trial. Horm Res Paediatr 90(1): 44-53.

5. Child CJ, Quigley CA, Cutler GB, Moore WV, Wintergerst KA, et al. (2019) Height gain and safety outcomes in growth hormone-treated children with idiopathic short stature: Experience from a prospective observational study. Horm Res Paediatr 91(4): 241-251.

6. Tao S, Li G, Wang Q, Hu Y (2015) Efficacy, and safety of human growth hormone in idiopathic short stature. Indian J Pediatr 82(7): 625-628.

7. Hou L, Liang Y, Wu W, Lin HH, Luo XP, et al. (2020) Comparison of the efficacy and safety of recombinant human growth hormone in treating idiopathic short stature and growth hormone deficiency in children. Growth Horm IGF Res: 53-54.

8. Child CJ, Zimmermann AG, Chrousos GP, Cummings E, Deal CL, et al. (2019) Safety outcomes during pediatric GH therapy: Final results from the prospective GeNeSIS observational program. J Clin Endocrinol Metab 104(2): 379-389.

9. Sävendahl L, Cooke R, Tidblad A, Beckers D, Butler G, et al. (2020) Longterm mortality after childhood growth hormone treatment: the SAGhE cohort study. Lancet Diabetes Endocrinol 8(8): 683-692.

10. Moher D, Shamseer L, Clarke M (2015) Preferred reporting items for systematic review and meta-analysis protocols (PRISMA-P) 2015 statement. Syst Rev 4(1): 1-9.

11. Downs SH, Black N (1998) The feasibility of creating a checklist for the assessment of the methodological quality both of randomized and non- randomised studies of health careinterventions. J of Epidemiology and Community Health 52(6): 377-384.

12. Rhie YJ, Yoo JH, Choi JH, Chae HW, Kim JH, et al. (2019) Long-term safety and effectiveness of growth hormone therapy in Korean children with growth disorders: 5-year results of LG Growth Study. PLoS One 14(5): e0216927.

13. Shaikh AAl, Daftardar H, Alghamdi AA, Jamjoom M, Awidah S, et al. (2020) Effect of growth hormone treatment on children with idiopathic short stature (ISS), idiopathic growth hormone deficiency (IGHD), small for gestational age (SGA) and Turner syndrome (TS) in a tertiary care center. Acta Biomed 91(1): 29-40.

14. Counts DR, Silverman LA, Rajicic N, Geffner ME, Newfield RS, et al. (2015) A 4-year, open-label, multicenter, randomized trial of Genotropin $®$ growth hormone in patients with idiopathic short stature: Analysis of 4-year data comparing efficacy, efficiency, and safety between an individualized, target-driven regimen and standard d. Horm Res Paediatr 84(2): 79-87.

15. Kim J, Suh BK, Ko CW, Lee KH, Shin CH, et al. (2018) Recombinant growth hormone therapy for prepubertal children with idiopathic short stature in Korea: a phase III randomized trial. J Endocrinol Invest 41(4): 475483.

16. Ying YQ Hou L, Liang Y, Wu W, Luo XP, et al. (2018) Efficacy and safety of recombinant human growth hormone in treating Chinese children with idiopathic short stature. Growth Horm IGF Res 42(43): 80-85.

17. Hwang JS, Lee HS, Lee KH, Yoo HW, Lee DY, et al. (2018) Once-weekly administration of sustained-release growth hormone in korean prepubertal children with idiopathic short stature: A Randomized, controlled phase II study. Horm Res Paediatr 90(1): 54-63.

18. Imane B, Myriam R, Maité T, Emmanuel P, Anne P, et al. (2018) Growth hormone in combination with leuprorelin in pubertal children with idiopathic short stature. Endocr Connect 7(5): 708-718.

19. Jung MH, Suh BK, Ko CW, Lee KH, Jin DK, et al. (2020) Efficacy and safety evaluation of human growth hormone therapy in patients with idiopathic short stature in Korea - A Randomized controlled trial. Eur Endocrinol 16(1): 54-59. 